\title{
15 ans de progrès en coloproctologie...et l'aventure continue pour la revue Côlon \& Rectum !
}

\author{
Y. Panis \\ (C) Lavoisier SAS 2015
}

C'est amusant car l'année dernière, en janvier, j'avais déjà commis cet éditorial de rentrée avec tout ce que j'espérais pour la nouvelle année. 2014 donc. En relisant cet éditorial je me rends compte que rien ou presque ne s'est réalisé ! Dans le désordre j'avais en effet souhaité : un meilleur salaire mais j'ai l'impression que la grille de la fonction publique est rouillée depuis quelques années ; toujours pas non plus de voiture de fonction....; ah oui, je me souviens! J'avais aussi rêvé d'un congrès des JFHOD avec plein de chirurgiens invités? Toujours pas non plus.... Bref, une vraie catastrophe ces souhaits.

Cette année, fini ! Plus de risque! Plutôt qu'à nouveau espérer des choses qui sont du domaine de la science fiction, pourquoi ne pas se retourner vers les 15 années qui se sont écoulées depuis l'an 2000 pour analyser les changements et les avancées observées dans notre belle spécialité médicochirurgicale qu'est la coloproctologie ? Au moins, aucun risque d'être déçu, car non seulement comme vous le verrez dans ce numéro forcément un peu spécial, les progrès ont été majeurs, mais en plus, c'est du passé. Donc aucun risque de se tromper...Forcément....

Bref, je ne vais pas vous énumérer ici l'ensemble de ces progrès majeurs qui ont révolutionné la prise en charge des patients de notre spécialité. A vous de vous promener dans les différents chapitres que vous a concocté le comité de rédaction. Nous abordons en effet plein de domaines comme le traitement médical et chirurgical des maladies inflammatoires de l'intestin, les troubles fonctionnels intestinaux, les troubles de la statique rectale, la cancérologie colorectale, l'anatomopathologie, la proctologie et j'en oublie sûrement. Mais la relecture de l'ensemble de ces textes rédigés par les rédacteurs de Côlon \& Rectum me fait dire que les progrès ont été observés dans tous les domaines.

Je ne prendrais qu'un seul exemple qui me tient à cœur et qui est celui évidemment de la chirurgie colorectale. Le 31 décembre 1999, quand j'avais devant moi au bloc opératoire une patiente de 78 ans avec un cancer du côlon droit : elle avait une préparation colique préopératoire (scandale !), une colectomie par laparotomie (honte !), et enfin vu son âge, même s'il existait une atteinte ganglionnaire, elle n'avait probablement pas de Folfox adjuvant (consternation !) car de toutes les façons, je ne l'avais pas présentée en RCP et j'avais pris cette décision tout seul dans mon coin (crime !). En résumé, tout a changé ! Et ce qui est vrai pour la chirurgie, l'est aussi pour tous les domaines de la coloproctologie médicale et instrumentale. Bref, nous avons vécu 15 ans de progrès constants.

Vous conviendrez donc que ce n'est pas le moment de baisser les bras. Pour cette raison, en changeant d'éditeur (Lavoisier maintenant), la revue Côlon \& Rectum repart de plus belle. Bonne année à tous ! Et à dans 15 ans pour un nouveau bilan!

\footnotetext{
Y. Panis $(\bowtie)$

Service de Chirurgie Colorectale,

Pôle des Maladies de 1'Appareil Digestif,

Hôpital Beaujon - Assistance Publique des Hôpitaux

de Paris (APHP), Université Paris VII (Denis Diderot),

100 boulevard du Général Leclerc, 92118 Clichy cedex

e-mail : yves.panis@bjn.aphp.fr
} 